causes as these, what reason is there for refusing to acknowledge that less evident forms of bronchial enlargement, or enlargement of the thymus, will very likely lead to similar symptoms, more or less evident according to the amount of disturbance produced in this or that individual case? If I have gone a step further, and attribute even such a condition as coma to similar causes, apart from the evidence which seems to justify this, I want pointedly to push home this conclusion by so doing ; that if, as a result of mediastinal enlargement, we admit one form of nervous explosion in the shape of peripheral disturbance, such as I have narrated, there is no reason for refusing to admit the occurrence of more profound central explosions in occasional cases. And some corroborative evidence $I$ would advance in favour of this is derived from that group of cases where a comatose condition ensues upon surgical interference with the pleural cavity. There are many explanations of the sudden death or coma attending upon interference with the pleura in cases of empyema. They cannot be discussed here; but the one which appears to my mind the most satisfactory is, that a central encephalic disturbance is produced through the influence of the peripheral branches of the pneumogastric.

One great obstacle to the acceptance of the view that I have been supporting here has been that its advocates have let it run away with them, and have offered bronchial enlargement as an explanation of all forms of paroxysmal dyspnœa. Whooping-cough, laryngismus stridulus, crowing respiration, spasmodic asthma, have all been explained in this way. I may disclaim any such notions for myself; and, indeed, I think that a careful consideration of the symptoms of individual cases proves conclusively that no one of these symptoms is due to any constant cause. Thus some cases of laryngismus are part of a central epileptic tendency; others are due to peripheral irritation in all sorts of places; and others, perhaps, to some local muscular fault in the larynx, as Riegel thinks. Then, too, it is said that so many cases of bronchial enlargement occur without the manifestation of any paroxysmal dyspnœa, that something besides the mere glandular swelling is wanting to lead to the production of the symptom. But here, again, no two cases are quite alike, either in the exact seat of the disease, in the direct implication of the nerves, or in the reacting circulatory troubles; and any one of these may just settle the question between paroxysmal dyspnœa or not. I am, however, inclined to think that a tendency to hasty nervous discharge predisposes to its occurrence, as might be expected, under slighter peripheral abnormalities. I am referring now more particularly to the frequency with which crowing respiration occurs in children who have actual convulsions.

3. What is the mechanism of the production of this paroxysmal dyspnœa? If I say that peripheral excitement leads to central stimulus and to nervous discharge, I only state what is every day before us as we live and move and have our being, and is illustrated sufficiently often in practice, where fits and dentition, or intestinal disturbance, are found associated. But I want to go further than this; because, in the first place, these cases of paroxysmal dyspnœa are not always entirely paroxysmal ; they are always paroxysmally exaggerated ; but there is, I think, in many, though all writers seem to deny this, a persistentslight, it may be-inspiratory sniffle for weeks or months. It seems to me that Brown-Séquard's explanation of certain genito-urinary cases helps us here; and that we have to do with a reflex paralysis or impairment of function about the medulla oblongata and pons Varolii, greater or less according to circumstances; greater in the case of the comatose state before alluded to; less in the simple inspiratory dyspnœea; but both the one and the other merely extremes in a chain of which we see the many links in a long series of cases of varying severity.

I suppose that, as the result of the abnormal stimulus, some erratic nerve-discharge is effected, or some circulatory disturbance is thereby engendered, leading to arterial spasm, which, if complete, as in some cases, leads to sudden death ; if less complete, may cause coma, or convulsion, or slight enervation and paresis of peripheral muscles.

Then, as to the seat of the local muscular fault; it is generally described as being in the glottis; no doubt correctly in many cases; but if it were so, in the desperate cases of which I have given instances, tracheotomy would get below a mere glottic spasm, and would relieve them, at any rate temporarily. But it does not; and then, moreover, in these cases, not unfrequently-and I think Rilliet and Barthez corroborate this - there is a considerable want of free expansion of the lung, even in the slighter cases, where there is not much evidence of glottic spasm; and I am therefore inclined to think that the condition is one of spasm of the whole lung, closely allied to what we suppose to be the case in spasmodic asthma.

4. The two points that I want to bring before your notice with regard to treatment are these. - $a$. As has just been said, tracheotomy is of no use; not for the reasons generally assigned, but because the real obstruction is quite as much in the whole lung as in the glottis itself. If this be the case, large doses of beHadonna-and, in children, by large doses I mean thirty drops of the tincture, repeated frequently, if necessary-are most likely to relieve such cases, or, perhaps, nitrite of amyl or other muscular relaxors. $b$. I have expressed my belief that some of these cases are cases of over-feeding; and yet, in nearly all cases, we fly to cod-liver oil and good living, and, in doing so, may make things worse. I beg to offer for your consideration and discussion that some of these cases may advantageously be starved rather than fed; and I am quite sure, with reference to tabes mesenterica, an allied condition, of course, that the most spare diet possible is the only treatment which in many cases suffices to stop the diarrhoea and keep the child in comparative health. The lymph-streams or chyle-streams are choked, and the lacteals cannot absorb; hence the continual passage of undigested food, and the constant diarrhoea. Reduce the supplies till the block is relieved, and, I believe, a cure may sometimes be effected.

5. Of prognosis nothing need be said. The cases themselves afford the indications, when coupled with the remarks already made on the treatment. But with regard to diagnosis, a good deal has been said by some as to the possibility of diagnosing the disease by careful percussion over the sternum or in the vertebral grooves. Some have even gone so far as to say that, in a large number of cases of whoopingcough, they find by this means that the bronchial glands are enlarged. I have gone into this question somewhat carefully amongst my outpatients, and, as a result, I believe it to be quite impossible, in the greater number of cases even of considerable or great enlargement of the mediastinal glands, to make out any increase of size by percussion. I have made it out once or twice, and found myself wrong on the post mortem table; so that probably, in many other cases also in which I had supposed it present, it was not so. To say this reduces the number of cases actually diagnosed by percussion to very narrow limits. Percussion will help us occasionally, but not often; and practically we shall have to form an opinion from the character of the dyspnœa, the aspect of the patient, and other points which arise in any particular case. Lastly, cases have been described in which all the symptoms of implication of the thoracic branches of the pneumogastric have been present, in which the symptoms speedily disappeared under stimulant and antispasmodic treatment; and it has, therefore, been supposed by some that a functional disturbance, with symptoms similar to those of organic disease, may exist. Such a case is given by Dr. Habershon in his Lumleian Lectures on the Pneumogastric Nerve, delivered last year at the Royal College of Physicians.

\section{THE ETIOLOGY OF ENTERIC FEVER.}

By SHIRLEY F. MURPHY, M.R.C.S.Eng., Medical Officer of Health, St. Pancras.

While Dr. Collie's able paper on the Etiology of Enteric Fever is still fresh in the memories of the readers of the BRITISH MEDICAL JOURNAL, I would ask them for a short time to consider the subject from the view-point of one who does not altogether agree with the theory entertained by the author concerning the manner in which enteric fever is communicated from the sick to the healthy.

I do not wish here to discuss the question whether enteric fever can arise from any condition of ordinary filth, but will assume, with Dr. Collie, that a specific poison is necessary for its production, and that each case of the disease requires for its existence an antecedent case.

Dr. Collie has stated as his opinion that certain cases which arose at the Homerton Fever Hospital among the attendants resulted from exposure to fresh enteric stools; he, however, adds, "We do not mean to say that cases do not arise from decomposing or decomposed stools, or from emanations from the lung and skin".

The question, so far as the attacks of attendants on the sick are concerned, is consequently limited to an inquiry into the possibility of these attacks arising from: 1. Conditions relating to the patient only, such as infection from breath, skin, and fresh stools; and, 2. Conditions relating to the hospital plus the patient. Now it may undoubtedly be assumed that, at Homerton, reasonable care is taken to dispose of the evacuations of a patient when they are passed, and that the stools are not kept in the ward for a longer time in this hospital than in another. If, therefore, the same precautions in this respect be taken in different hospitals, the number of attacks among the attendants, due to the first class of causes, must have some relation to the number of susceptible persons exposed to infection, for it must be remembered that the first class of causes is constantly operative. That is, there are always skins, breath, and fresh stools for the nurses to be exposed to ; and, on the other hand, the second class, due to the hospital as well as to the enteric stools, will only be in force under certain conditions; such conditions, perhaps, as lead to the escape of air from the enteric stool-infected drain into the 
lavatory or ward; attacks due to this class will vary in different hospitals out of all proportion to the number of attendants.

Now Dr. Collie admits that both these classes of causes are sufficient for the production of enteric fever ; and it becomes, therefore, necessary to ascertain how far each class may be responsible for the attacks of attendants. Should the first class be responsible at all, it is more likely to be responsible for by far the greater number of the attacks from the reason that it is constant in its action.

I hope to show that this is not the case, and for this purpose shal take the statistics of the London Fever Hospital in Liverpool Road for the last twenty-four years, seeing that, since 1854 only, enteric and typhus fevers have been distinguished from each other in the records of this hospital. I would point out that, under any circumstances, there must be a greater incidence of attacks on the attendants in the enteric fever wards than on the attendants in other wards, inasmuch as there is necessarily a large amount of enteric fever-stools in the drains of the former; but I wish to show that, if the attacks known to be due to conditions special to the hospital be eliminated from the total, so few remain unaccounted for, that it is impossible to attribute them to the first set of causes, which, as already pointed out, must be constantly in operation.

The following table shows the number of enteric fever patients admitted into the London Fever Hospital, and the number of attendants attacked in each year.

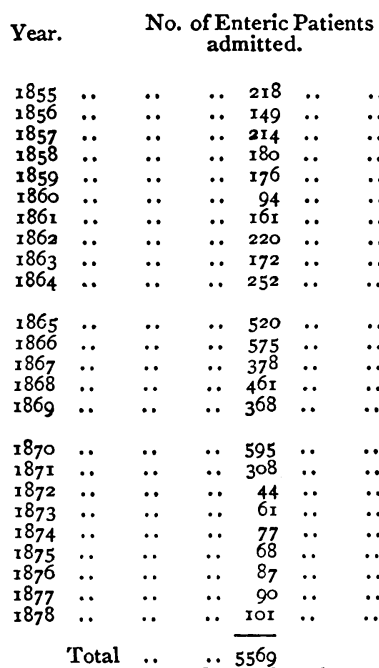

Attacks from Enteric Fever among Officers, Nurses, Servants, and other Patients not suffering from Enteric Fever when admitted.

. Two house-servants attacked No attacks

. ", ,

Öne scarlet fever patient attacked No attacks

O’ne nurse attacked

. No attacks

Cook, porter's child, assistant medical officer, and one patient attacked No attacks

. Two nurses attacked

Two housemaids attacked

No attacks

One laundry-maid and one kitchen maid attacked.

Two nurses attacked

One nurse attacked

.. No attacks

$\because ", \quad$,

$\because "$,

- ",

O’ne nurse and one patient attacked

It will be seen from the above table that a certain number of person engaged in the service of the hospital have been attacked by enteric fever, but it is necessary to consider how far these were related to the patients in the hospital, before the possibility of their illness being due to the first or second group of causes can be determined.

In 1855 , two housemaids, who had no connection whatever with the wards or patients, and who lived in a separate house, were attacked.

In 1859 , one scarlet fever patient, who had no connection with the enteric fever ward, was attacked.

In 1862 , one nurse was attacked.

In 1864, the cook, the porter's child, an assistant medical officer, and one patient contracted enteric fever.

With reference to the cases in 1862 and 1864 , I cannot do better than quote from the hospital report on the subject.

"For three years, the cases of enteric fever, and the cases which have not been true fever, have been kept together; 644 cases of the former and 1,108 of the latter have thus been treated together in the same wards, interspersed among one another. Not one of the patients in these wards has contracted enteric fever. The nurses and attendants throughout the hospital have enjoyed a like immunity. During the last nine years, upwards of $\mathbf{I}, 500$ cases of enteric fever have been under treatment in the hospital, but only one nurse has suffered from it, and in her case it was doubtful if the attack did not originate out of the hospital. These considerations made it the more extraordinary when four cases of enteric fever originated in the hospital, in the spring of the past year, at a time when there were scarcely any patients in the building suffering from this form of fever. Two of the persons attacked were the cook and the porter's child, who lived in separate buildings a third was an assistant medical officer, and a fourth was a patient lying in a ward as distant as possible from that used by the enteric fever patients. The assistance of the Medical Officer of Health for Islington was called in, and important defects in the drainage of the hospital were discovered, which were at once remedied. No case of enteric fever occurred in the hospital after that, notwithstanding the unusually large number of cases admitted during autumn."

In 1866, two nurses were attacked.

Concerning these cases, the hospital report, referring also to three cases of cholera occurring among the nurses, states: "The cause of this (cholera) outbreak was at once inquired into by the medical officers, and it was discovered that a drain, into which the cholera evacuations were thrown, had become blocked up. This was cleaned out on the 2nd of September, and a system of flushing commenced; the result was that, from that date, no fresh case (of cholera) occurred. But, what was not a little remarkable, within a short time of this outbreak, an interval not longer than the period of incubation of the disease, two nurses were attacked with enteric fever, a disease, so far as its causes are concerned, presenting many points of resemblance to cholera, and like it, rarely spreading in hospitals. No other case of enteric fever occurred in the hospital; and the circumstance is the more remarkable from the fact that, during the last five years, the cases of enteric fever, and the cases which have not been true fever at all, have been kept together in the same wards; that 1,739 cases of the former, and 2,123 of the latter, have thus been interspersed among one another, and yet that none of the patients, and, before September 1866 , none of the nurses and attendants in these wards, have contracted enteric fever."

In 1867 , two housemaids, who were in no way related to the enteric fever patients or the wards, and who lived in a separate house, were attacked.

In 1869, one laundrymaid, who had no communication with the patients except that she washed their linen, and one kitchen-maid, who lived in a separate house, and had no communication with the patients, were attacked.

In 1870 , two nurses were attacked.

In 187.1 , one nurse was attacked.

In 1878 , one nurse employed in the enteric fever ward was attacked; and, at the same time, a patient who was convalescing from scarlet fever, but who had left the hospital, was also attacked with enteric fever. With regard to these, I would point out that the scarlet and enteric fever wards adjoin each other, and that the drain from the lavatory of the one ward immediately joins the lavatory drain of the other; so that three feet from the lavatories there is but one drain common to the two wards. The fact of the nurse in the enteric fever ward, and the patient in the scarlet fever ward, being attacked simultaneously, is sug. gestive of the cause being operative on both wards, and this could only take place through drainage.

Since 1854 , therefore, 5,569 patients suffering from enteric fever have been admitted into the London Fever Hospital, and nineteen persons engaged in the hospital have been attacked with enteric fever. Of these, ten were in no way connected with the enteric fever patients or the enteric fever wards. Of the other nine, one was a laundry-maid, whose duties would bring her into contact with the soiled linen of the patients, but who otherwise was not in contact with the patients. Of the remaining eight, there were special circumstances connected with drainage which would probably account for four, leaving four for whose attacks there is no explanation given.

Seeing, then, that some cause of attacks among attendants other than contact with fresh enteric stools was in operation over the whole hospital-and this is clearly shown by the fact of ten attendants suffering from enteric fever who were in no way connected with the enteric fever patients or wards-it is not to be expected that those who were engaged in these wards should enjoy a greater immunity than other attendants in the hospital. There is every reason for expecting a greater incidence of attacks on the attendants in enteric fever wards than on others, even supposing these attacks to be produced only by the second class of causes.

I have already pointed out that special hospital conditions are sufficient to account for the illness of four of the eight attendants in the enteric fever wards who were attacked. There are, therefore, four remaining; and I need hardly ask what is the probability of only four persons being attacked from causes of the first class continuously in action in twenty-four years? Even supposing the whole eight were due to causes of the first class, how does this compare with the number of attacks at the Homerton Fever Hospital? In six years and a half, during which this hospital is used for fever patients, seventeen nurses and three laundry-maids are attacked with enteric fever, sixteen of these nurses being engaged in attendance on acute, one on convalescent, enteric fever patients. I regret I am unable to state the number of nurses engaged in the enteric fever wards in the London Fever Hospital during the twenty-four years under consideration; and I am 
willing to admit that probably the proportion of nurses to patients in past days in this hospital was somewhat smaller than would be now the case in the Homerton Fever Hospital with our more modern notions of nursing; but I would point out that the large number of patients in the former institution at least warrants the opinion that no fewer nurses each year were engaged in the enteric wards of the London Fever Hospital than of the Homerton Fever Hospital. The two hospitals are therefore roughly comparable. If, then, the Homerton Fever Hospital were to maintain the number of attacks among attendants it has hitherto done, it would have had in twenty-four years nearly sixty of its nurses attacked, as compared with eight during the same period in the London Fever Hospital. With regard to a possible objection that many nurses in the latter institution must, before 1854, have suffered from enteric fever, and that thus a staff of insusceptible nurses had been created, I would state that the nurses at the London Fever Hospital have, as at most special hospitals, remained but a short time in its service; and this fact has been so well known that, having regard to the risks incurred in nursing typhus, the subject has been constantly under the consideration of the Committee of this institution. It may therefore be reasonably asked, why do nurses at the Homerton Fever Hospital contract enteric fever seven times as frequently as at the London Fever Hospital ? Can this possibly be due to causes which must be as much in operation at Liverpool Road as at Homerton - such as the skin, breath, or fresh stools of enteric fever patients? or is it not due to some difference in the structure of the hospitals themselves? It is needless to state that, with regard to cubic space allotted to each patient, ventilation of wards and lavatory arrangements, the Homerton is in no way inferior to the London Fever Hospilal; but, with regard to the drainage, the greatest difference exists.

Dr. Collie has, however, naturally asked-If the drains be to blame, how comes it that other patients in the enteric fever wards who had not suffered from enteric fever escaped? This appears at first to be a strong argument; but when the relative exposure of a nurse to the drains of sinks and sluice-pans, compared with the exposure of a patient to water-closets during the short period of convalescence is considered, it is evident that an attack of a patient would be an exceptional occurrence. Moreover, remembering the fact that such a patient, if attacked, would probably not become ill until after leaving the hospital, it is not surprising that there is a want of evidence that such patients have been attacked.

I do not, however, wish at the present time to do more than show that the risk to nurses in the enteric fever wards at the Homerton Fever Hospital is at least seven times the risk to nurses similarly engaged in the London Fever Hospital; and that there is every reason to think that the few attacks in the latter institution are due to a condition special to the hospital itself, and not to a cause which must be continuously in operation. I would therefore submit that the theory of infection from the breath, skin, and fresh stools of enteric fever patients is not supported by the frequency of attacks of nurses in the Homerton Fever Hospital.

There can be no doubt that the greatest possible care is required in the arrangement of drains and drain ventilation in a fever hospital, and the subject is deserving of the attention of all who have charge of such institutions. An important lesson might be learnt by a careful consideration of the varying drain conditions in different fever hospitals, and of the number of attendants who are attacked with enteric fever.

\section{TYPHOID FEVER.}

By THOMAS J. WALKER, M.D., Surgeon to the Infirmary, Peterborough.

THE discovery that the infecting medium of typhoid fever is located in the stools of a patient suffering from the disease has already been productive of great results, and surely is destined to benefit mankind still further. The fact is admitted alike by those who, with Sir T. Watson, Budd, etc., believe that no case of typhoid can arise except from germs originally derived from the excreta of a previous patient, as well as by those who, with Murchison, Niemeyer, etc., believe in the possibility of an independent origin of typhoid fever in putrifying filth. It has formed the basis of every successful struggle with typhoid epidemics; and any evidence brought forward with the view of throwing professional opinion back to the position occupied in the last decade, when the contagionists could say no more than that typhoid was contagious, like small-pox, scarlet fever, etc., must be carefully examined. In the JourNAL for March 8th are published two papers on the etiology of enteric fever, having for their avowed object the establishment of views on the contagious nature of this disease differing from those so ably expounded by Dr. Murchison, and endorsed by such men as Drs. Wilks, Andrew, Ogle J. W. Balfour, W. T. Gairdner, Muirhead, and many others who have, in writing, expressed to me within the last six months their view that enteric fever is not contagious in the usual sense of the word, as well as by almost every other hospital physician in Great Britain. The first of these papers is an instalment of a communication in which Dr. Collie gives a clear and doubtless perfectly fair statement of the sanitary condition of the Homerton Fever Hospital, and of certain cases of enteric fever which have originated in it during the time that it has been opened. As this paper is still incomplete, any criticisms on Dr. Collie's deductions must be postponed, but the facts which he has already brought forward appear strongly to favour the pythogenic theory of the origin of the disease. The second is written by Dr. W. Thomson, and I regret that to his statements the terms which I have applied to those of Dr. Collie do not equally apply. Having myself watched the epidemics and seen many of the cases on which Dr. Thomson professes to base his conclusions, I am able to throw some light on the evidence on the faith of which he asks the leaders of our profession to change the views on which they found their practice of mixing typhoid patients with others in hospital, and otherwise disregarding any necessity for isolation. The first epidemic to which he refers is that which occurred in 1874 , in which his recollection leads him to state that he had at one time under his own charge two hundred and thirty-five cases ; ${ }^{*}$ this being the epidemic which, as he says, induced him to publish a popular pamphlet on Infectious or Catching Diseases for the "aidance" of the ignorant public, who had not been taught to believe typhoid "highly contagious". Singularly enough, the epidemic here selected afforded most convincing proof of the mode in which enteric fever actually spreads. As pointed out at the time in Dr. Thomson's reports as medical officer of health, the fever, though occurring to a certain extent in other parts of the town, was really epidemic only in a suburb situated about a mile from the rest of the city. The town generally was supplied with drinking-water from the shallow wells to which I shall presently refer; but this suburb had water laid on from the river (a sluggish stream)-the water being drawn from a point within one hundred and thirty yards of which three drains discharged into the same river the sewage of about six thousand of the inhabitants. There was incontestable proof that the fever depended on the impure water-supply; and happily Dr. Thomson, as medical officer of health, adopted this view, and joined with others in urging the proprietors of this suburb to supply its inhabitants with purer water, and to make other sanitary changes, which, without any attempt at isolation or quarantine, checked the epidemic then, and have since almost banished enteric fever from that part of the town, where previously it had for many years been more or less endemic. From the water-main to this suburb a branch-pipe was laid to one establishment only in the town, and to this I have been the family attendant for twenty years. Year after year, cases of typhoid fever occurred among the inmates, chiefly young persons sleeping together two or three in a room; but $\mathbf{I}$ never had the slightest reason to suspect that any case arose from contagion. As the cases recurred, various half-measures of sanitary reform were adopted by the proprietors; but it was not until three years since. that I was able to secure the actual cutting off of the supply of riverwater from every part of the establishment, excepting one tap at the side of the kitchen-fire. Since that date, only one case of typhoid has: occurred; and the managers of the establishment, who had for economical reasons always resisted my demand for a radical change in the water-supply, triumphantly adduced the fact that the kitchen-maid had typhoid as a proof that the fever "was caught somewhere or was in the

* Everything tending to show the value of evidence brought forward in a controversy is of importance, and I therefore feel it right to say that I doubt the accuracy of Dr. Thomson's recollection on this point. I have carefully looked through all his pub lished reports, as medical officer of health for this year; and, although I find that he refers to the prevalence of typhoid fever in the suburb referred to in the text, there is no scription of such an awful epidemic as would be implied by the fact that two hundred and thirty-five persons (that is, taking $\mathrm{Dr}$. Thomson's estimate of the population and

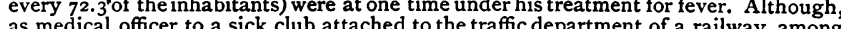
as medical officer to a sick club attached to the traffic department of a railway, among whose servants and their families this fever specially prevailed, Dr. Thomson may have had during this epidemic more cases than fell to the lot of other medical men, should say that, on the very humblest estimate, at least an equal number of cases must be given to the whole body of the profession in the town, including the surgeon to the locomotive club of the same railway, the dispensary and parish surgeons. Cal culating thus, there must have been at one time one in every thirty-six of our population down with fever-a calamity which must have arrested the attention of the whole country, and which could hardly have been kept such a profound secret until now, when Dr. Thomson announces the fact on which this calculation is based. In his report, dated September 26th, 1874, he gives the total number of deaths from typhoid for the quarter during which this outbreak prevailed as eight; and, although willing to credit Dr. Thomson, the rest of my medical brethren here, and myself, with extraordinary skill, I can hardly believe that, in an epidemic in which four hundred and seventy cases of fever were under treatment at one time, we could escape with such a result. 